\title{
Influence of channel reservation on the handover calls flow in mobile network
}

\begin{abstract}
Aleksandar Lebl ${ }^{1}$, Dragan Mitićc ${ }^{2}$ Žarko Markov² Vladimir Matić$^{1}$
In this paper the properties of handover traffic are analyzed in the system with channel reservation. The reservation of several traffic channels only for handover calls leads to probability of handover calls congestion decrease to the level of per mill or even lower. The congestion of all traffic channels in the neighbouring cells is the cause that handover traffic process deviates from the Poissonian distribution and this deviation is negligible, because handover calls congestion probability is very low. This low handover calls probability is also the reason why implementation of one equivalent cell instead of 6 neighbouring cells surrounding the central cell does not model satisfactory the traffic flow in the mobile network. The randomness of handover calls arrival to the central cell is tested by our originally developed simulation program. Coefficient of variation of time between handover calls arrival to the central cell is calculated as the result of simulation. We introduced the ratio of the coefficient of variation of the time between new handover calls appearance in the central cell to the value of this coefficient for surrounding cell as the measure of handover calls randomness: the nearer this ration to 1 , the more random is handover calls arrival to the central cell.
\end{abstract}

K e y w or ds: handover traffic, Poissonian process, neighbouring and central cells, simulation program

\section{Introduction}

Limitation of usable frequency spectrum and energy savings, among other reasons, led to the development of mobile networks with small cells. These cells inevitably cause a high number of handovers of existing connections [1]. Handover calls loss is considered very undesirable and many techniques are developed which decrease probability of handover calls loss [2]-[7]. One common measure is channel reservation for handover calls that reduces the handover calls loss at the expense of primary calls loss increasing. That is the reason why it is growing the interest to study the process of handover calls in detail. The dilemma whether creating handover calls is Poissonian process is resolved in [8], but in the model without serving priority for handover calls. However, in practice it is much more often the case that the handover calls have priority. This paper shows how much the process of handover calls birth deviates from the Poissonian one if reservation of channels provides priority to handover calls and whether this deviation affects common calculation method.

\section{Model, assumptions and designations}

It is usual to model the cellular network as a group of cells with the same characteristics: dimensions, number of channels and users, call birth rate. These assumptions allow service quality calculation. The cell dimension is determined by the radius $R$. The speed of movable users is designated by $v$ and handover probability by $P_{h}$. The number of channels in each cell is designated by $N$ and the number of users in the cell is significantly higher than the number of channels. It means that it is possible to apply Erlang model, whose characteristic is that call congestion is equal to time congestion. The primary call rate in each cell is designated by $\lambda_{\mathrm{p}}$ and handover call rate from the network into the considered cell is designated by $\lambda_{\mathrm{h}}$.

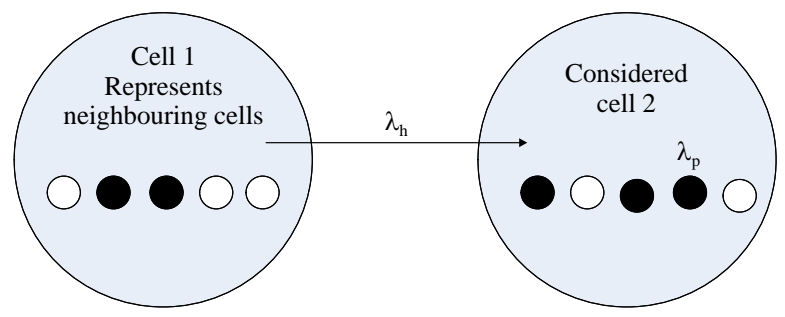

Fig. 1. The simplified model of mobile telephony network: neighbouring cells are replaced by equivalent cell 1

The usual presentation of network influence on the considered cell is presented on the Fig. 1. The cell 1 which models the network is idealized, $i e$ it is supposed that it has the same influence on the considered cell 2 as all neighbouring cells together. In the model handover calls from all neighbouring cells enter the cell 2 over the cell 1 . The handover calls serving has the priority and it is adopted in this paper that handover call may be lost only if all channels are busy, ie blocked. As handover call loss is very undesirable event, the dimensioning of resources is performed in such a way that handover call loss is significantly lower than the primary call loss. It is

\footnotetext{
1 Department of Radio Communications Engineering, Institute IRITEL a.d., Belgrade 11080, Serbia, lebl@iritel.com, vmatic@iritel.com, ${ }^{2}$ Department of Optical Communications, Institute IRITEL a.d, mita@iritel.com, zmarkov@iritel.com
} 
convenient that handover calls loss, if it exists, is in the range of per mills.

Of course, the opposite assumption about the influence in the network is also valid, ie that the cell 2 has influence on the neighbouring cells over the cell 1 .

The channels are presented as circles in the Fig. 1: uncoloured circles correspond to idle channels and black circles correspond to busy channels. The probability of state $j(=0,1, \ldots, N)$ in the group of sources (channels) in one cell is designated by $P_{\mathrm{j}}$ and it presents the probability that $j$ sources are busy. The equality

$$
P_{N}=B=B_{\mathrm{h}}
$$

is valid in the case that all channels are busy and it is important to point out that this probability has very low value, because it determines handover calls dropping. The probability that all channels are busy in $i(=1,2,3,4,5,6)$ cells is designated by $P\{i\}$.

The primary calls are generated according to Poissonian model: the number of primary calls in the unit of time $\left(\lambda_{\mathrm{p}}\right)$ has the same value regardless of the serving system state. Therefore, the value of $\lambda_{\mathrm{p}}$ is the same as if any number of channels is busy $(0,1,2, \ldots, N-1, N)$.

\section{The defined calculation models}

There are several methods of call handling which give priority to handover calls serving over primary calls serving. These methods are: (1) some number of traffic channels reservation only for handover calls; (2) handover calls waiting in a queue for serving and (3) the bit-rate decreasing in already realized connections to handle handover traffic. The first method is the most often implemented one and is more detailed analyzed in the available literature than methods 2 and 3 . The number of reserved channels may be fixed or it may be dynamically altered according to traffic load [9]. The applied schemes for channel assignment are presented in more detail in [10] and, besides pure reservation of some channels to handover calls, these schemes are: (1) admission of a new call with a certain probability depending on the number of busy channels; (2) division of traffic channels in two groups: the first one for common use of both calls and the second one only for handover calls; (3) limitation of the number of new generated calls.

Generally speaking, according to [11] in some cases it is enough to consider only a single cell to model the traffic behaviour in a mobile system with a number of cells. Such an analysis is suitable when new arrivals rate is identical in all cells and the handover arrivals and departures rate are also equal in all cells. The multicell model is reserved for the case when there are some specific points with the significantly higher traffic rate or when user moving routes are not homogenous (handover rate between cells is variable).

\section{The process of primary and handover calls generation}

The main difference between Poissonian and handover calls flow is in the state when all channels are busy. If all channels in the cell 2 are busy (Fig. 1.), primary calls continue to come on serving. On the contrary, if all channels in the equivalent cell 1 are busy, new handover calls may not enter the cell 2 because they come from other cells using idle channels of neighbouring cells. As the cell 1 from Fig. 1 represents all neighbouring cells, the question is: what means that all channels are busy in the cell 1? We shall present all neighbouring cells (NC1-NC6) by their channels to get answer to this question, Fig. 2.

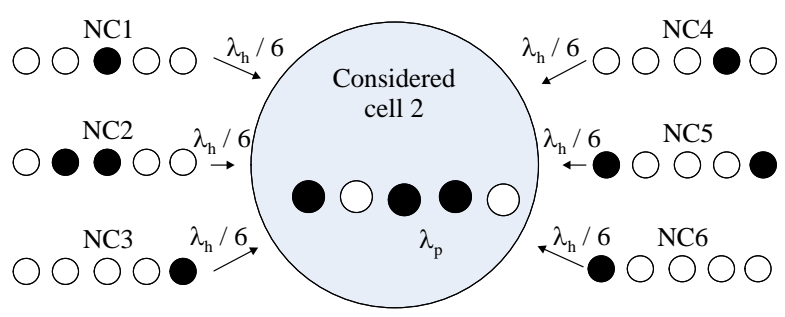

Fig. 2. The real model of the mobile telephony network part: central cell enclosed by 6 neighbouring cells

According to the assumption, all cells are identical. The handover calls flow from each neighbouring cell is Poissonian, but its rate is equal to one sixth of the total rate. If all channels are busy in some neighbouring cell (NC1 and NC5, Figure 3), there is no handover calls flow from these cells. The probability that all serving sources (ie channels) in one cell are busy is $P_{N}=B=B_{\mathrm{h}}$. This is also the probability that there is no handover calls flow from this cell. Therefore, the total calls flow from any neighbouring cell equals $\lambda_{\mathrm{h}}(1-B)$ [8]. Handover calls flow in the case that all channels are busy in $i(=1,2,3,4,5,6)$ neighbouring cells is

$$
\lambda_{h i}=\frac{\lambda_{h}}{6} \cdot(6-i)
$$

and this event probability is

$$
P(i)=B_{h}^{i} \cdot\left(\left(1-\left(B_{h}\right)\right)^{6-i}\right.
$$

Let us consider the handover calls flow as the random variable. It is discrete random variable which may have 7 values $(i=0,1,2,3,4,5,6)$.

The states when all channels are busy in one or more neighbouring cells are called blocking states. In this state the following events are distinguished:

1. All channels are busy only in one cell. The probability of this event is $B_{h} \cdot\left(1-B_{h}\right)^{5}$. The handover calls flow is $\lambda_{h 1}=(5 / 6) \cdot \lambda_{h}$;

2. All channels are busy only in two neighbouring cells. The probability of this event is $B_{h}^{2} \cdot\left(1-B_{h}\right)^{4}$. The handover calls flow is $\lambda_{h 2}=(4 / 6) \cdot \lambda_{h}$. 


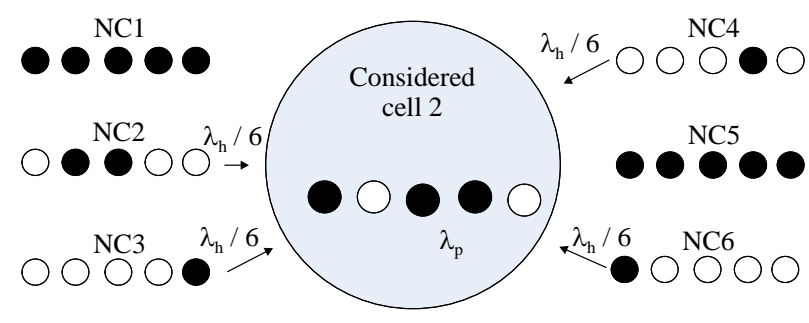

Fig. 3. Real model of the mobile telephony network without idle traffic channels in neighbouring cells 1 and 5 (NC1 and NC5)

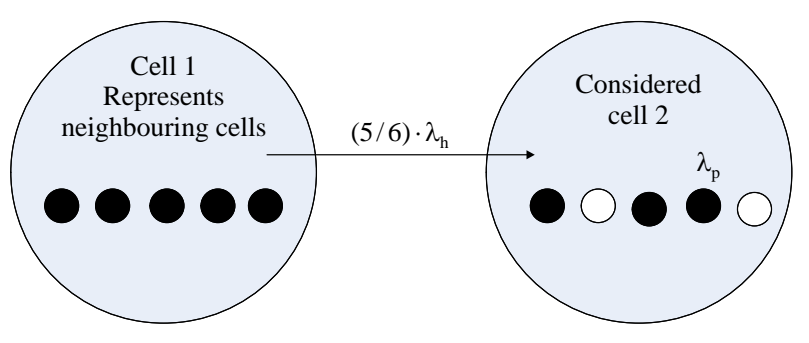

Fig. 4. Simplified model of mobile telephony network part without idle traffic channels in one of neighbouring cells
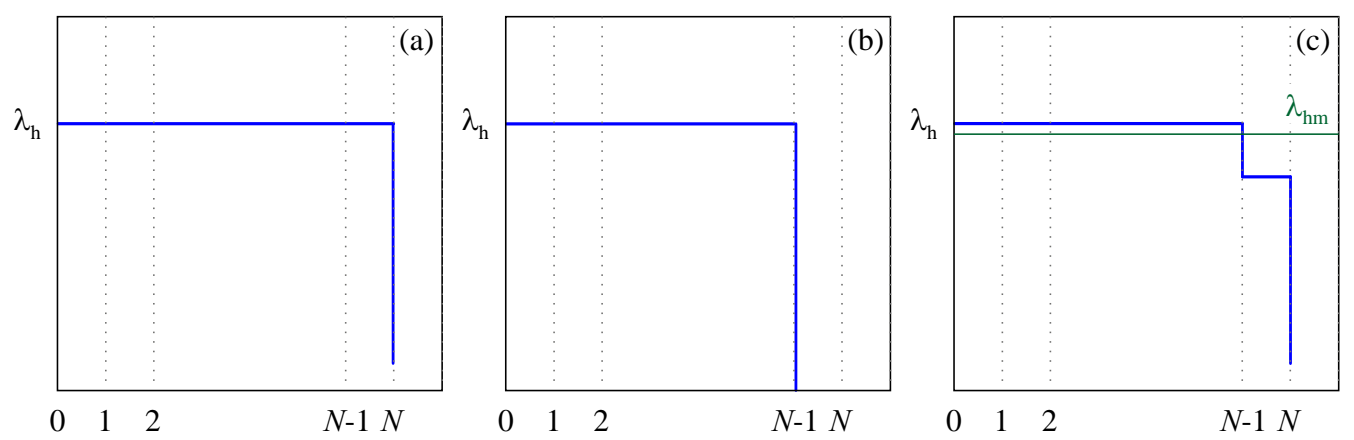

Fig. 5. Poissonian calls flow: (a) - ideal case; (b) - in the simplified model according to Fig. 1 and Fig. 4 , (c) - in real model according to Fig. 2 and Fig. 3

3. All channels are busy only in three neighbouring cells. The probability of this event is $B_{h}^{3} \cdot\left(1-B_{h}\right)^{3}$. The handover calls flow is $\lambda_{h 3}=(3 / 6) \cdot \lambda_{h}$.

4. All channels are busy only in four neighbouring cells. The probability of this event is $B_{h}^{4} \cdot\left(1-B_{h}\right)^{2}$. The handover calls flow is $\lambda_{h 4}=(2 / 6) \cdot \lambda_{h}$.

5 . All channels are busy only in five neighbouring cells. The probability of this event is $B_{h}^{5} \cdot\left(1-B_{h}\right)^{1}$. The handover calls flow is $\lambda_{h 5}=(1 / 6) \cdot \lambda_{h}$.

6 . All channels are busy only in six neighbouring cells. The probability of this event is $B_{h}^{6}$. There is no handover calls flow $\left(\lambda_{h 6}=0\right)$. If there are idle channels in all neighbouring cells, the handover calls flow is $\lambda_{h 0}=\lambda_{h}$, and the probability of this event is

$$
P\{0\}=1-\sum_{i=1}^{6} P\{i\}
$$

The process characteristics in all states are similar, but call inflow rate is variable. Let us determine the mean value of this rate.

The mean value of random variable handover calls inflow rate $\lambda_{h m}$ is

$$
\lambda_{h m}=\sum_{i=0}^{6} \lambda_{h i} \cdot P\{i\}
$$

The mean value of handover calls inflow rate may be calculated for the value $B_{h}=0.01$ (which is unrealistic high value for handover calls congestion) and it is $\lambda_{h m}=$ $0.998389 \cdot \lambda_{h}$. It may be said that mean handover calls rate is not changed if channel reservation methods are implemented for handover calls.
The probabilities of events 2 to 6 may be neglected, because the value of probability $B_{h}$ is very low. That is why handover calls rate in the state of congestion is practically always very near to $(5 / 6) \cdot \lambda_{h}$, Fig. 4 .

We may distinguish three models of handover calls flow and notice the difference between these three cases of the Poissonian flow of handover calls:

- Figure 5(a) presents ideal Poissonian call flow which is the same in all system states;

- Figure 5(b) presents Poissonian handover calls flow rate which does not exist in the congestion state of equivalent cell representing the network;

- Figure 5(c) presents Poissonian handover calls flow rate which is modelled in this paper as a real case.

Symbolic representation of handover calls flow rate as a function of time is sketched in Fig. 6 .

It is obvious that handover calls flow rate is closer to ideal one in real case when all neighbouring cells are considered than if network is modelled by one cell. This is proved by the simulation program which comprises simulations for each cell separately, as described in Section 6 . The parts of simulation program, which correspond to one cell, are presented in [7]. The results of simulation program in [7] are verified by their comparison to the calculation results.

\section{Examples}

Let us consider the network formed of cells whose radius is $R=10 \mathrm{~km}$ and the mean users velocity is $v=60 \mathrm{~km} / \mathrm{h}$. The number of traffic channels is $N=5$, the offered traffic is $A_{p}=2 E$ and the number of channels reserved for handover calls is $r=2$. It is calculated that

$$
P_{h}=0.17
$$




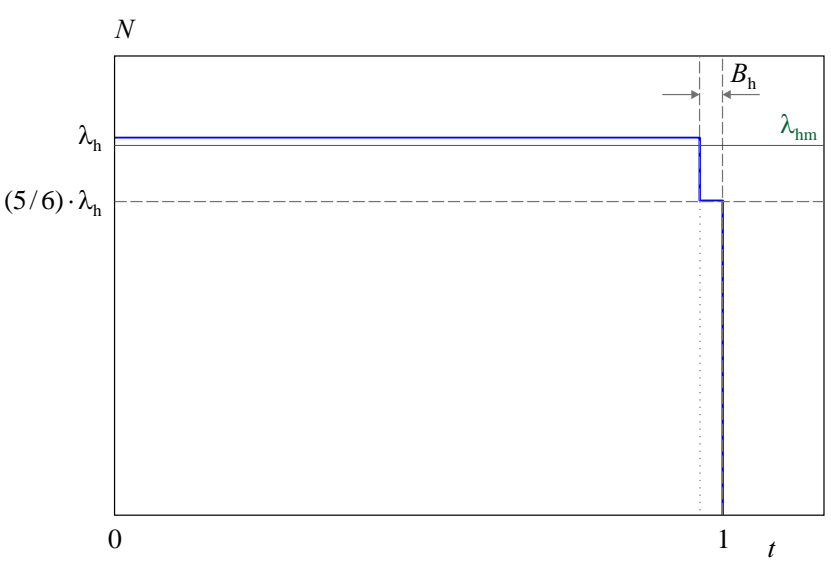

Fig. 6. Symbolic presentation of handover calls flow rate as a function of time

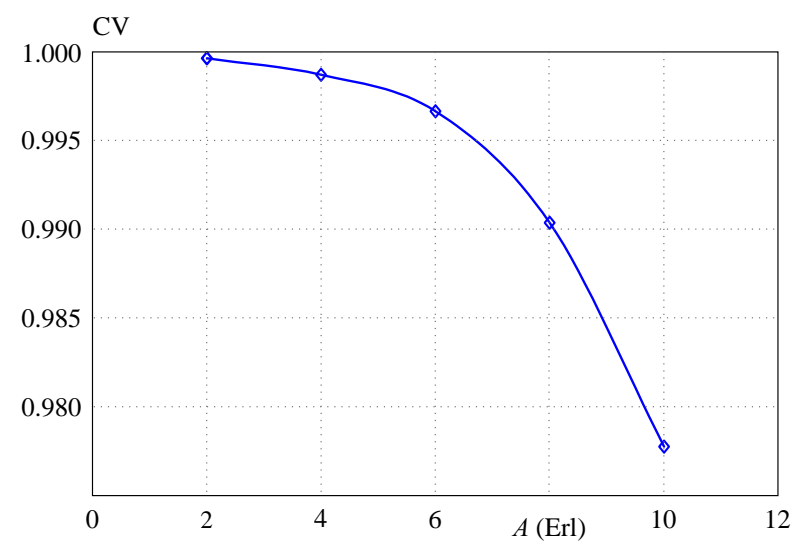

Fig. 8. Coefficient of Variation of handover calls $(\mathrm{CV})$ as a function of offered traffic $\left(A_{p}\right)$ in a system with $N=14, r=3$ channels and $P_{h}=0.17$

[12], [7]. The probability of primary traffic loss is $B_{p}=$ 0.269 and the probability of handover traffic loss is $B_{h}=$ 0.00111 in this case.

The probability of handover calls rate decrease for one sixth in the case that one of six neighbouring cells is congested equals $B_{h}=0.00111$. The probability of higher handover calls rate decrease is, in the best case, $B_{h}^{2}=$ $0.00111^{2}=0.00000123$. As traffic loss in the same time designates also time congestion, the time period while all traffic channels are busy in two neighbouring cells is $4.4 \mathrm{~ms}$ in the busy traffic hour. The mean time interval between consecutive calls in this case is several tens of seconds. That is why, as a consequence, the congestion time ie the time of reduced calls flow is negligible and without any influence on the characteristics of handover calls generation.

\section{Numerical example 2}

Let us consider the network with the same dimensions ( $R=10 \mathrm{~km}$ and the same users' mean velocity $(v=60$ $\mathrm{km} / \mathrm{h}$. The number of channels is $N=14$, the offered traffic is $A_{p}=10 \mathrm{E}$ and the number of reserved channels for handover calls is $r=3$. As $R$ and $v$ are the same as in the example 1 , it is $P_{h}=0.17$ again. The offered traffic of $10 \mathrm{E}$ in the group of 14 channels causes traffic loss $5.6 \%$ in standard Erlang model. In the model with two traffic components and channel reservation the primary calls loss

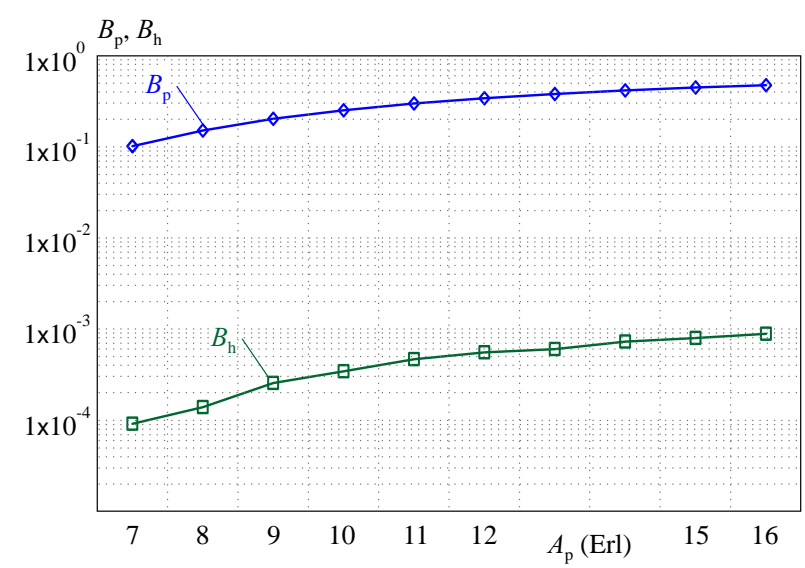

Fig. 7. Primary and handover calls congestion as a function of offered traffic for the system where it is $N=14, r=3, P_{h}=0.17$

is $B_{p} \approx 0.25$ and the probability that all traffic channels are busy $i e$ the probability of handover calls loss is $B_{h} \approx$ 0.0004. The probability that all traffic channels are busy in two or more network cells is negligible, ie without any influence. The handover calls inflow to the considered cell is very similar to the Poissonian process (with the probability $\approx 0.9996$ ).

It is important to emphasize that the influence of handover calls congestion in neighbouring cells (if channel reservation is applied) practically does not depend on the traffic load. The probability that all traffic channels are busy in the neighbouring cells, $B_{h}$, is negligible even at unreal high offered traffic values $A$, as may be concluded from Fig. 7. That is why the statement about approximately the same value of handover calls rate is valid for all traffic values.

Verification of handover calls randomness in the simulated traffic process is not simple. Namely, Coefficient of Variation $(\mathrm{CV})$, which is defined as the ratio of standard deviation and mean value of random variable [13] is always equal 1 for Poissonian distributed continual random variable. In the simulation process time is not continual variable, but a discrete one. Simulation process may be considered as a series of Bernoulli Trials (BT) according to [14]. Events designated as Successes and Failures may be distinguished in these BT. If handover call generation is treated as a success, then the time between two events (successes) may be described by geometric distribution. $\mathrm{CV}$ at geometric distribution depends on the probability, $i e$ it is not a constant number as at Poissonian distribution. That is why it is necessary to find a new method to compare process of handover calls flow into the considered cell, Fig. 3 with Poissonian process.

Coefficients of Variation of handover calls arrival into the considered (central) cell (CV7) and for all 6 surrounding cells (CV1-CV6) are measured in a simulation model as the one in the Fig. 3. The mean value CVm is calculated for 6 surrounding cells. The handover calls arrival into the surrounding cells is a reliable real time simulation of a Poissonian process. It is possible to say that the comparison of values $\mathrm{CV} 7$ and $\mathrm{CVm}$ is an indicator whether the process of handover calls arrival into the considered 


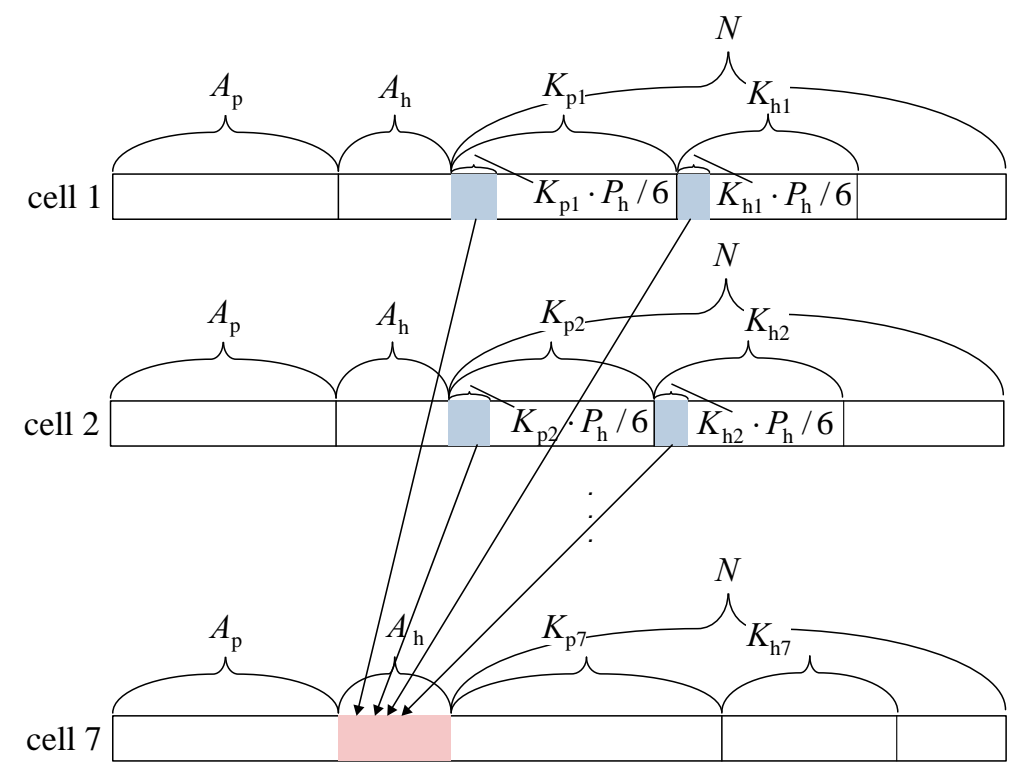

Fig. 9. The procedure for events generation in the simulation process

cell may be also supposed to be a reliable simulation of the Poissonian process.

The graph on the Fig. 8 proves that handover calls process arrival into the considered cell is the same as the one generated in all other cell if the losses are negligible, $i e$ it is the reliable simulation of Poissonian process. In the case of increased traffic load/losses some of primary calls from the surrounding cells may not become handover calls. It is the reason why the similarity with the Poissonian process is a bit modified.

\section{Simulation}

As already stated, simulation program is based on the similar simulation of the system with handover calls, which is presented in detail in [7]. The program version from [7] is modified in such a way that it realizes the simulation for 7 cells of mobile telephony network in the same time ( 1 central cell and 6 surrounding cells), in accordance to the model presented in the Fig, 3. The first addition to the program from [7] is generation of one more random number with uniform distribution (RN2) whose value determines the ordinal number of the cell (among total 7 cells) for which the following simulation step is intended. The simulation step is performed as presented in [7].

Binding events realized in one simulation step to the generated random number may be explained using Fig. 9 . The whole set of generated random numbers is transformed to the range $\left(0, A_{p}+A_{h}+N\right)$, where $A_{p}$ is the offered primary calls traffic, $A_{h}$ is the offered traffic of handover calls, and $N$ the number of available traffic channels. Besides, $K_{p}$ and $K_{h}$ in Fig. 9 are instantaneous number of channels engaged by primary and handover calls. The second modification to the process of generating the event in the case that it is a random number intended for one of first 6 cells refers to hatched range of numbers, whose size is $K_{p} P_{h} / 6$ and $K_{h} P_{h} / 6$, (whereas $P_{h}$ is handover probability). Therefore, if the random number is in the range $\left(A_{p}+A_{h}, A_{p}+A_{h}+K_{p} P_{h} / 6\right)$ or $\left(A_{p}+A_{h}+K_{p}, A_{p}+A_{h}+K_{p}+K_{h} P_{h} / 6\right)$, the established call will not finish. Instead of that, this call will be transferred to the central, seventh cell, thus simulating handover. In such a way handover calls are generated in the seventh cell for the range of numbers $\left(A_{p}, A_{p}+A_{h}\right)$.

Let us now suppose that RN2 determines that the next simulation step has to be realized in the cell 7 and that, after that, the following generated random number is in the range $\left(A_{p}, A_{p}+A_{h}\right)$, which corresponds to a new handover call start. We have already pointed out that handover calls for the seventh cell in the simulation procedure are formed from a part of the primary and part of the handover call in the cells $1,2, \ldots, 6$ at the time of their leaving the cell $1,2, \ldots, 6$. That is, why no event (ie new call) is generated in the seventh cell if the random number for the seventh cell falls in the range $\left(A_{p}, A_{p}+A_{h}\right)$.

\section{Conclusions}

The main conclusion is that channel reservation for handover calls contributes that the process of handover calls inflow is practically equated to the Poissonian flow. The main reason for handover calls deviation from the Poisson flow is the states of handover calls congestion. Deviations from the Poisson flow with respect to the state without congestion are very small. In addition, in a wide range of traffic load, the states of handover calls congestion are short so that they have very low influence on the deformation of the handover calls Poisson flow. The examples in this paper also prove that multicell model is more satisfactory when mobile system performances 
are analyzed in the case of uniform traffic load per cells than if the influence of neighbouring cells is replaced by one equivalent cell. One additional important paper contribution is that it gives the measure of the extent to which handover calls generation nature is random. It is proved on the simulation program results basis that this process is random for small values of offered primary traffic (the ratio between the coefficient of variation of time between handover calls for the central cell (CV7) and the mean value of these coefficients for surrounding cells $(\mathrm{CVm})$ is constant and equal 1$)$. This randomness is slowly degraded when offered traffic is increased (the ratio CV7/CVm very slightly decreases below 1 ).

\section{REFERENCES}

[1] B. Jabbari, "Teletraffic aspects of evolving and next-generation wireless communication networks", IEEE Personal Communications, vol. 3, no. 6, pp. 49, December 1996, DOI: 10.1109/98.5564 73.

[2] J. V. Agustina, J. V. P. Yhang, and R. Kantola, "Performance evaluation of GSM handover traffic a GPRS/GSM network", Eighth International Symposium on Computers and Communications ISCC 2003, pp. 137-142, Finland, June-July 2003, ISBN: 0-7695-1961-X.

[3] A. A. Adewale, S. N. John, and E. R. Adagunodo, "Performance Comparison of Dynamic Guard Channel Assignment with Buffered Prioritized Scheme for Mobile WiMAX Network", Proceedings of the World Congress on Engineering 2016 Vol I WCE 2016, pp. 1-5, June 29th Jule 1st 2016, London, ISBN 978-988-19253-0-5, ISSN: 2078-0958.

[4] A. E. Xhafa and O. K. Tonguz, "Dynamic Priority Queueing of Handover Calls Wireless Networks: An Analytical Framework", IEEE Journal on Selected Areas Communications, vol. 22, no. 5, pp. 904-916, June 2004, DOI: 10.1109/JSAC.2004.826927.

[5] R. Beraldi, S. Marano, and E. Palumbo, "Analysis of new priority queueing strategies for handoff and originating calls mobile cellular radio systems", IEEE Conference: Wireless Communications System Symposium, pp. 63-69, 1995., DOI: 10.1109/WCSS.1995.588484.

[6] T. Thumthawatworn, A. Pervez, and P. Santiprabhob, "Enhanced Adaptive Traffic Dependent Handover Decision System for Wireless Mobile Networks, International Journal on Advances Networks and Services, vol. 7, no. 1 \& 2, pp. 118-129, 2014, ISSN: 1942-2644.

[7] D. Mitić, A. Lebl,Ž. Markov, and V. Kosjer, "Determination of the Traffic Properties of Cells with Mobile Users Using a Mixed Traffic", Radioengineering, vol. 28, no. 4, pp. 801-814, December 2019., Proceedings of Czeck and Slovak Technical Universities, ISSN 1210-2512, DOI: 10.13164/re.2019.0801.

[8] E. Chlebus and W. Ludwin, "Is Handoff Traffic Really Poissonian?", Proceedings of ICUPC'95, pp. 348-353, 1995, DOI:10.11 09/ICUPC.1995.496919.

[9] A. A. Adewale, S. N. John, and E. R. Adagunodo, "Performance Comparison of Dynamic Guard Channel Assignment with Buffered Prioritized Scheme for Mobile WiMAX Network", Proceedings of the World Congress on Engineering 2016, Vol I WCE 2016, pp. 1-5, June 29th July 1st 2016, London, ISBN 978-988-19253-0-5, ISSN: 2078-0958.

[10] Y. Fang and Y. Zhang, "Call admission control schemes analysis wireless mobile networks", IEEE Transactions on Vehicular Technology, vol. 51, no. 2, pp. 371-381, March 2002.
[11] G. Ming and Z. Pan, Advances Wireless Networks: Performance Modelling, Analysis and Enhancement, Nova Science Publishers, Inc., 2008, ISBN: 1-60021-713-3., Chapter 13: J. Martinez-Bauset, V. PLA, D. Garcia-Roger, et al Admission Control Policies to Minimize Blocking/Forced-Termination Multimedia Cellular Networks.

12] M. Schwartz, Mobile wireless communications, Cambridge University Press, 2005, DOI: https://doi.org/10.1017/CB097805118 11333.

[13] V. B. Iversen, Teletraffic Engineering and Network Planning, Denmark: Technical University of Denmark, 2015.

14] W. Feller, An Introduction to Probability Theory and Its Applications, third edition, 1968.

Received 5 August 2020

Aleksandar Lebl was born in Zemun, Serbia, in 1957. He received his BSc and MSc from the Faculty of Electrical Engineering in Belgrade, Republic of Serbia, in 1981. and 1986, respectively, and his PhD from the Faculty of Technical Science in Novi Sad, in 2009. He was employed from 1981 in the Switching Department of Institute for Electronics and Telecommunications IRITEL in Belgrade. During years he worked on the project of Digital Switching System for Serbian Telecommunication Industry. From 2015. he works in Radio communications department, also in IRITEL.

Dragan Mitić was born in Belgrade, Serbia, in 1953. He received his BSc and MSc from the Faculty of Electrical Engineering in Belgrade, Republic of Serbia, in 1977 and 1984, respectively, and his $\mathrm{PhD}$ from the Faculty of Technical Science in Novi Sad, in 2002. Dr Mitić is scientific counsellor in IRITEL, Institute for Electronics and Telecommunications, Belgrade, Serbia. From 1977. until 1989. he was employed at the Land Forces Military Technical Institute in Belgrade, and since 1989. in IRITEL. Dr Mitić is author or co-author of more than 200 international and national scientific and professional papers. He works on several research projects for equipment of specific applications.

Žarko Markov was born in Žitište, Serbia, in 1946. He received his BSc, MSc and PhD from the Faculty of Electrical Engineering in Belgrade, Republic of Serbia, in 1969., 1975. and 1976., respectively. Dr Markov is a scientific counsellor in IRITEL, Institute for Electronics and Telecommunications, Belgrade, Serbia. Area of work: Switching technics, Teletraffic theory, Network signalling. Author or co-author of more than hundred papers and six books. At the University of Belgrade, School of Electrical Engineering, Dr. Markov was a professor at the course of Switching technics and Network signalling.

Vladimir Matić was born in Belgrade, Serbia, in 1963. He received his BSc and MSc from the Faculty of Electrical Engineering in Belgrade, Republic of Serbia, in 1989, and 1994, respectively, and his PhD from the Faculty of Technical Science in Novi Sad, Serbia, in 2016. From 1996 he is employed in Radio communications department of Institute for Electronics and Telecommunications IRITEL in Belgrade, where now holds the position of project leader. He published more than 50 scientific papers in international and national journals and conferences and participated in realization of several scientific and research projects of the Ministry of Science and Technology of Republic of Serbia and Institute IRITEL.

Received 5 August 2020 